\title{
Surgical treatment of giant mesenteric fibromatosis presenting as a gastrointestinal stromal tumor: a case report
}

\author{
Christos N Stoidis ${ }^{1 *}$, Basileios G Spyropoulos², Evangelos P Misiakos², Christos K Fountzilas ${ }^{3}$, Panorea P Paraskeva ${ }^{4}$, \\ Constantine I Fotiadis ${ }^{2}$
}

\begin{abstract}
Introduction: Intra-abdominal fibromatosis, usually located at the mesenteric level, is a locally invasive tumor of fibrous origin, with no ability to metastasize, but a tendency to recur. Certain non-typical cases of intra-abdominal fibromatosis with involvement of the bowel wall can be misdiagnosed because of their different biological behavior.
\end{abstract}

Case presentation: We describe the case of a 64-year-old Caucasian man presenting with mesenteric fibromatosis and involvement of the bowel wall, who was treated surgically. The macroscopic and microscopic appearance of the lesion mimicked a gastrointestinal stromal tumor, a tumor with potential malignant behavior.

Conclusion: It is essential to make an early and correct diagnosis in such equivocal cases, so that the appropriate treatment can be chosen and suitable patients admitted to clinical trials if appropriate. New and reliable criteria for discriminating between intra-abdominal fibromatosis and gastrointestinal stromal tumor should be proposed and established because novel sophisticated therapeutic strategies have been introduced in the international literature.

\section{Introduction}

Mesenteric desmoids account for less than $10 \%$ of sporadic desmoid tumors and are particularly common in patients with familial adenomatous polyposis (FAP) [1]. Of these tumors, $70 \%$ are intra-abdominal, and most of these involve the mesentery [1]. The association between desmoid tumors and FAP is particularly strong in patients with Gardner's syndrome [2]. Patients with FAP and a family history of desmoid tumors have a $25 \%$ chance of developing a desmoid tumor [2].

Gastrointestinal stromal tumors (GISTs) on the other hand originate from gastrointestinal pacemaker Cajal cells, which are the primary effectors controlling gut motility [3]. GISTs may develop to a large size and usually present with bleeding. Radiologically, the tumor usually contains a central ulceration caused by necrosis from outgrowth of its blood supply. GISTs may grow into the organ lumen, remain entirely on the serosal

\footnotetext{
* Correspondence: aris.80@hotmail.com

'Department of Surgery, Athens Navy Hospital, 70 Deinokratous Street,

11521, Athens, Greece

Full list of author information is available at the end of the article
}

surface, or even become pedunculated within the abdominal cavity. Spread is by direct invasion or bloodborne metastases. Computed tomography (CT) scans provide useful information about the extent of extraorgan spread.

The enigmatic biology and anatomic location of intraabdominal fibromatosis (IAF) highlight the need to discriminate between these two diseases. IAF is benign and exclusively locally aggressive, whereas GISTs present with a risk of aggressive clinical behavior, depending on location, diameter and number of mitoses; thus GISTs are potentially malignant and may lead to distant metastases. The fact that IAF and GISTs have different biological behaviors makes treatment recommendations difficult despite the recent introduction of new therapeutic strategies. A significant factor limiting any attempts at generalizing management strategies is the small number of cases available for analysis, reflecting the relative rarity of the disease.

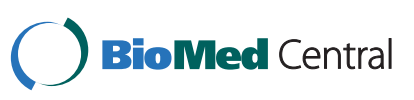

C 2010 Stoidis et al; licensee BioMed Central Ltd. This is an Open Access article distributed under the terms of the Creative Commons Attribution License (http://creativecommons.org/licenses/by/2.0), which permits unrestricted use, distribution, and reproduction in any medium, provided the original work is properly cited. 


\section{Case presentation}

A 64-year-old Caucasian man was admitted to our hospital with a ten-year-history of a mild diffuse abdominal pain associated with anorexia. He reported no noticeable weight loss or other symptoms. His family history was unremarkable and he had no history of previous abdominal surgery.

On physical examination, we noted a mass in the left upper quadrant of the abdomen, which was mobile. A $\mathrm{CT}$ scan of the abdomen revealed a homogeneous, nonenhancing mass, $70 \times 100 \mathrm{~mm}$ in size, in the mesenteric region near the small bowel, with a consistency suggesting thick mucinous or proteinaceous material, which possibly represented an intestinal wall tumor. There were no other relevant findings.

At laparotomy, a solid mass measuring $80 \times 100 \times 120$ $\mathrm{mm}$ was identified at the root of the jejunal mesentery, infiltrating the adipose tissue and bowel wall, and in close association with the superior mesenteric and the middle colic vessels (Figure 1). The mesentery contained several large lymph nodes. A small amount of free peritoneal fluid was present. The mass was totally excised with a loop of jejunum and without apparent interference with the blood supply to the bowel. However, bowel ischemia did occur, and the patient required a second laparotomy three days later. The ischemic injury seemed to be secondary to venous obstruction. It was necessary to resect an additional segment of small bowel measuring $600 \mathrm{~mm}$ in length. A primary anastomosis was performed.

Our patient recovered well, and was discharged from the hospital one week after the second laparotomy. The final tissue diagnosis showed spindle-shaped fibroblasts with elongated nuclei and a benign appearance. The cut surface was tan, whorled, and firm, without necrosis, cystic change, or hemorrhage. Microscopy showed loosely arranged spindle cells with bland, oval nuclei

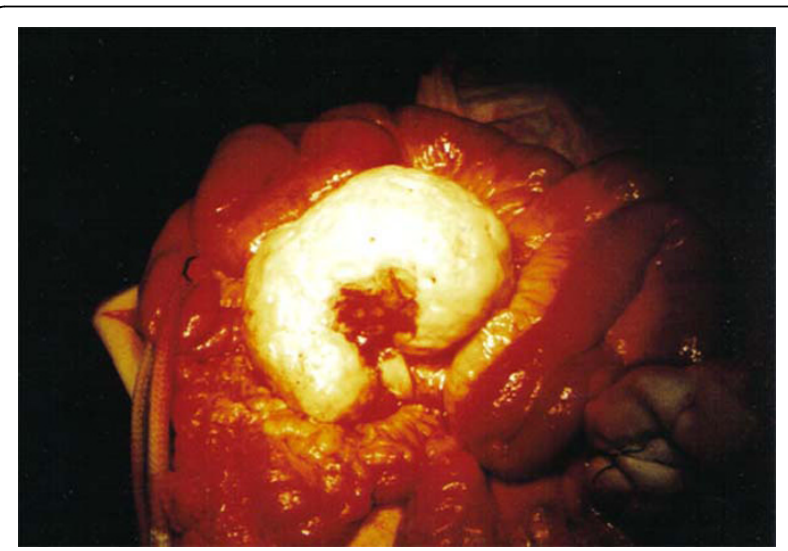

Figure 1 Surgical preparation. The resected tumor measured $120 \times 80 \times 100 \mathrm{~mm}$.

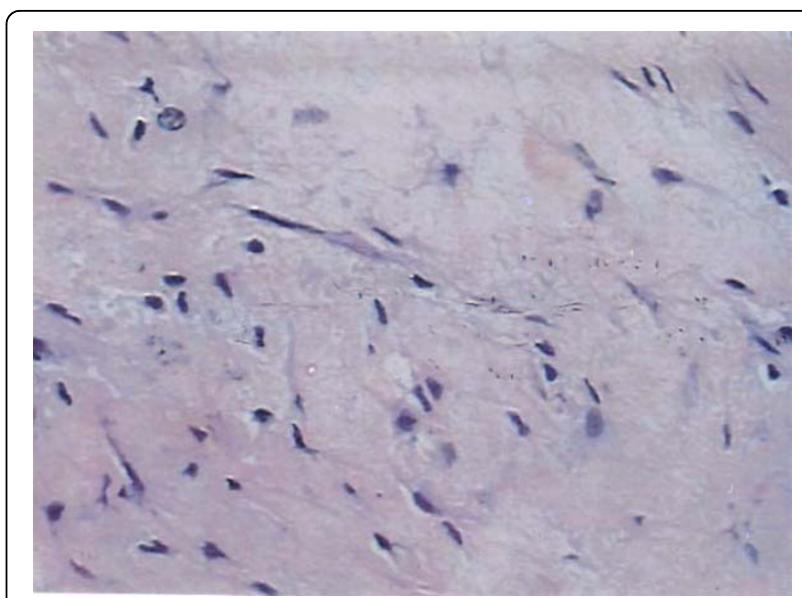

Figure 2 Spindle cells growing in sweeping fascicles, with eosinophilic cytoplasm.

and minimal cytoplasm (Figure 2). There were also plump spindle cells with tapering ends, with oval, vesicular nuclei and moderate amounts of eosinophilic cytoplasm. There were many thin-walled vessels of varying caliber. There were no cells with epithelioid features, and any inflammatory cells, calcification, osseous metaplasia, necrosis, or mitoses. The tumor had infiltrated the muscularis propria and had non-infiltrating margins. The tumor cells were negative for antibodies to CD117, S100, CD34, and smooth muscle actin, and positive for desmin. The small sample of peritoneal fluid was free of malignant cells.

A diagnosis of fibromatosis of the jejunum and mesentery was made. Six months after resection, a CT scan of the abdomen showed no evidence of residual or recurrent tumor. Our patient had no evidence of disease at follow-up 16 months after surgery. However, the clinical data and radiologic findings gave rise to a diagnostic dilemma: was the lesion an intermediate or high-risk malignant GIST originating from the bowel wall and spreading to the mesentery, or was this a benign lesion such as IAF originating from the mesentery and infiltrating the bowel wall?

\section{Discussion}

Intra-abdominal spindle cell lesions are uncommon, and often present a diagnostic challenge [4]. The differential diagnosis of a bland spindle cell tumor involving the gastrointestinal (GI) tract and the mesentery includes GIST, fibromatosis, and inflammatory myofibroblastic tumor [5].

IAF is the most common primary mesenteric tumor with spindle cell morphology [6]. Its biological behavior is intermediate between benign fibrous tissue proliferation and fibrosarcoma. Fibromatosis characteristically is locally invasive and tends to recur, but does not 
metastasize. Although mesenteric desmoid tumors tend to be aggressive, there is considerable variability in their growth rate during the course of the disease. In fact, the biology of intra-abdominal desmoid may be characterized by initial rapid growth, followed by stability or even regression. However, mesenteric desmoid, by virtue of its relationship to vital structures and its ability to infiltrate adjacent organs, may cause important complications, including intestinal obstruction, ischemia and perforation, hydronephrosis, and even aortic rupture $[7,8]$. Despite these complications, the overall ten-yearsurvival for patients with intra-abdominal desmoids can be as high as 60 to $70 \%$ [9].

In contrast to abdominal wall desmoids, surgery for intra-abdominal desmoid tumors is much more dangerous, and is associated with increased morbidity and mortality, mainly due to hemorrhagic complications or extensive enterectomy (caused by small bowel involvement or involvement of the base of the mesentery and major portions of the mesenteric blood supply) requiring long-term parenteral nutrition [10]. Additionally, a large percentage of patients with intra-abdominal desmoid tumors have unresectable disease [11]. In these cases, expectant treatment is favored, and biopsy should be preferred to excision. If clinical obstruction warrants operative therapy, then bypass, as opposed to major resection, is indicated, followed by a trial of medical therapy. Small bowel obstruction can occur in nearly half of patients with intra-abdominal desmoid tumors, and in $70 \%$ of these, diffuse, dense fibrotic adhesions are responsible [12]. Debulking has no place as a therapeutic measure, as it almost invariably leads to more aggressive and infiltrative desmoid growth [12]. Most authors consider surgery a reasonable first-line treatment for abdominal wall tumors, but it should be used as a last resort for intra-abdominal desmoid tumors, and only in specific circumstances (for example, when tumors cause major complications, do not involve vital organs and vessels on preoperative imaging, after failure of systemic pharmacologic therapy, or when surgery is the only possible therapeutic option, such as in the case of a rapidly growing tumor) [13]. Radical (free margin) excision offers the best chance for cure and of avoiding local recurrence [14]. Unfortunately, radical surgery is not always a straightforward procedure because of the extent and invasiveness of the tumor. Therefore, very high rates of recurrence (up to $88 \%$ ) should be expected [14]. Given the high likelihood of recurrence and prolonged survival even in the setting of advanced disease, some authors have suggested that a trial of watchful waiting along with minimally toxic agents such as sulindac and anti-estrogen therapy may be the best strategy, particularly in patients with minimal symptoms [15]. For clearly inoperable cases, cytotoxic chemotherapy, especially doxorubicin-based or low-dose vinblastin and methotrexate has been proposed [12].

The location of a desmoid tumor within the mesentery of the small bowel may complicate the management of patients with FAP and interfere with surgical strategy, preventing proctectomy or ilial pouch-anal anastomosis (IPAA), at least in some patients [15]. It may also make a diverting ileostomy impossible. Moreover, in this group of patients, recurrence rate after complete resection is particularly high [16]. It has been suggested that this increased risk may be due, at least in part, to the added manipulations of the mesentery during IPAA. Management of desmoid tumors in patients with FAP is further complicated by the fact that clinical course of the disease in this group is particularly variable and unpredictable, and may be disastrous. In a few highly selected patients with extensive desmoid tumors involving the mesentery, intestinal transplantation has been performed [17].

It is generally easy to diagnose primary IAF in its classic presentation as a mesenteric mass, because of its distinctive gross and microscopic features [18]. However, when it presents primarily as an intestinal wall tumor, as in our patient, the diagnosis of GIST must be seriously considered. For our patient, we considered GIST an unlikely diagnosis because of the whorled appearance on the cut surface of the tumor, and the histologic evaluation confirmed this, hence the diagnosis of fibromatosis of the jejunum and mesentery was established.

Histological features characteristic of GIST include the presence of spindle or epithelioid cells with variable architecture, mitotic activity and nuclear atypia, and myxoid or hyalinized stroma. Necrosis and hemorrhage can also be seen. By contrast, IAFs are characterized by a spatially homogeneous proliferation of wavy spindle cells without atypia, associated with collagen deposition (often of the keloidal type) and an infiltrative border. These features are sufficiently characteristic of mesenteric fibromatosis to allow distinction from GIST on the basis of routinely stained sections in most cases. In the few equivocal cases, immunohistochemical analysis can provide more precise diagnosis.

The distinction between these neoplasms is very important because they have different biological behaviors, and there are important clinical implications for the patient. Complete excision of the tumor with a margin of uninvolved tissue (when this is possible) is the most effective treatment yet described, and it may be followed by other therapies if GIST is diagnosed. After the more radical (free margin) resections required for GISTs, the five-year survival rate is approximately $45 \%$, whereas for metastatic disease it drops to $20 \%$ [19]. The tumor is resistant to radiotherapy, whereas radiation is controversial for intra-abdominal desmoid tumors 
$[18,19]$. Imatinib mesylate is an effective systemic agent and is indicated in patients with KIT (CD117)-positive gastrointestinal stromal tumors that cannot be surgically removed, and/or have spread to other parts of the body. Recent research indicated it may also be useful as part of the post-surgery adjuvant therapy for adult patients who have had their GISTs completely removed. The role of imatinib in the management of abdominal desmoid tumors remains unproven [20].

\section{Conclusion}

The optimum treatment protocol for desmoids tumors has not yet been established and, in many cases, a multidisciplinary approach including surgery, chemotherapy, and radiation therapy is required. The rarity of cases in even major oncological centers has traditionally limited the ability to study this disease. The notion that a specific genotype can predict the development of an aggressive desmoid tumor in a given patient could prove to be valuable in allowing appropriate patient selection for early therapy or even a chemopreventive strategy. Several novel pharmacologic and biologic treatment approaches are actively being developed, although longterm follow-up is needed for their substantiation.

The aim of this report is to stress the importance of correctly characterizing a tumor localized in the bowel wall and infiltrating the mesentery to plan the appropriate treatment because tumor diagnoses based on immunohistochemical staining or traditional histologic criteria alone are not specific enough.

\section{Consent}

Written informed consent was obtained from the patient for publication of this case report and accompanying images. A copy of the written consent is available for review by the Editor-in-Chief of this journal.

\section{Acknowledgements}

The authors state that there was no extra-institutional funding. We thank llias A. Kouerinis, who was a major contributor in composing the manuscript.

\section{Author details}

'Department of Surgery, Athens Navy Hospital, 70 Deinokratous Street, 11521, Athens, Greece. ${ }^{2}$ Third Department of Surgery, University of Athens Medical School, Attikon University Hospital, 1 Rimini Street, 12462, Chaidari, Greece. ${ }^{3}$ Department of Internal Medicine, Athens Navy Hospital, 70 Deinokratous Street, 11521, Athens, Greece. ${ }^{4}$ Second Department of Propaedeutic Surgery, University of Athens Medical School, Laikon General Hospital, 17 Agiou Thoma Street, 11527, Athens, Greece.

\section{Authors' contributions}

CIF was the patient's surgeon, and was involved in drafting the manuscript and critically revising it for important intellectual content. EPM, CNS, BGS, PPP and CKF have made contributions to conception and design. CNS contributed to the analysis and interpretation of data and wrote the paper. All authors read and approved the final manuscript. All authors contributed equally to the final draft of the manuscript. CIF has given the final approval of the version to be published.

\section{Competing interests}

The authors declare that they have no competing interests.

Received: 5 April 2010 Accepted: 23 September 2010

Published: 23 September 2010

\section{References}

1. Okuno S: The enigma of desmoid tumors. Curr Treat Options Oncol 2006, 7:438-43.

2. Church J, Lynch C, Neary P, LaGuardia L, Elayi EL: A desmoid tumorstaging system separates patients with intra-abdominal, familial adenomatous polyposis-associated desmoid disease by behavior and prognosis. Dis Colon Rectum 2008, 51:897-901.

3. Connolly EM, Gaffney E, Reynolds JV: Gastrointestinal stromal tumours. Br J Surg 2003, 90:1178-86.

4. Al-Nafussi A, Wong NACS: Intra-abdominal spindle cell lesions: a review and practical aids to diagnosis. Histopathol 2001, 38:387-402.

5. Colombo P, Rahal D, Grizzi F, Quagliuolo V, Roncalli M: Localized intraabdominal fibromatosis of the small bowel mimicking a gastrointestinal stromal tumor: a case report. World J Gastroenterol 2005, 11:5226-5228.

6. Rodriguez JA, Guarda LA, Rosai J: Mesenteric fibromatosis with involvement of the gastrointestinal tract. A GIST simulator: a study of 25 cases. Am J Clin Pathol 2004, 121:93-8.

7. Holubar S, Dwivedi AJ, O'Connor J: Giant mesenteric fibromatosis presenting as small bowel obstruction. Am Surg 2006, 72:427-9.

8. Collins D, Myers E, Kavanagh D, Lennon G, McDermott E: Mesenteric desmoid tumor causing ureteric obstruction. International J Urol 2008, 15:261-262.

9. Dozois EJ, Dozois RR: Desmoid disease. In Mayo Clinic Gastrointestinal Surgery. Edited by: Kelly KA, Sarr MG, Hinder RA. Saunders Eds: Philadelphia; 2004:563-565.

10. Pai SA, Zaveri SS: Intra-abdominal fibromatosis of the jejunum and mesentery. J Clin Pathol 2004, 57:1119.

11. Schlemmer M: Desmoid tumors and deep fibromatoses. Hematol Oncol Clin N Amer 2005, 19:565-71.

12. Latchford AR, Sturt NJH, Neale K, Rogers PA, Phillips RK: A ten-year-review of surgery for desmoid disease associated with familial adenomatous polyposis. Br J Surg 2006, 93:1258-64.

13. Rampone B, Pedrazzani C, Marrelli D, Pinto E, Roviello F: Updates on abdominal desmoid tumors. World J Gastroenterol 2007, 13:5985-5988.

14. Melis M, Zager JS, Sondak VK: Multimodality management of desmoid tumors: how important is a negative surgical margin? J Surg Oncol 2008, 98:594-602.

15. Sturt JNH, Clark SK: Current ideas in desmoid tumors. Familial Cancer 2006, 5:275-85.

16. Tamura $\mathrm{K}$, Tani M, Kinoshita $\mathrm{H}$, Yamaye $\mathrm{H}$ : Mesenteric desmoids tumor of the interposed jejunal pouch after total gastrectomy. World J Surg Oncol 2006, 4:27-31.

17. Chatzipetrou MA, Tzakis AG, Pinna AD, Kato T, Misiakos EP, Tsaroucha AK, Weppler D, Ruiz P, Berho M, Fishbein T, Conn HO, Ricordi C: Intestinal transplantation for the treatment of desmoid tumors associated with familial adenomatous polyposis. Surgery 2001, 129:277-81.

18. Sakorafas GH, Nissotakis C, Peros G: Abdominal desmoid tumors. Surg Oncol 2007, 16:131-142.

19. Yantiss RK, Spiro IJ, Compton CC, Rosemberg AE: Gastrointestinal stromal tumor versus intra-abdominal fibromatosis of the bowel wall. Am J Surg Pathol 2000, 24:947-957.

20. Mace J, Biermann JS, Sondak V: Response of extraabdominal desmoid tumors to therapy with imatinib mesylate. Cancer 2002, 11:2373-2379.

doi:10.1186/1752-1947-4-314

Cite this article as: Stoidis et al: Surgical treatment of giant mesenteric fibromatosis presenting as a gastrointestinal stromal tumor: a case report. Journal of Medical Case Reports 2010 4:314. 